\title{
Diffusion of published cost-utility analyses in the field of health policy and practice
}

\author{
Seema S. Sonnad \\ University of Pennsylvania \\ Dan Greenberg \\ Ben-Gurion University of Negev \\ Allison B. Rosen \\ University of Michigan \\ Peter J. Neumann \\ Harvard School of Public Health
}

Objectives: The diffusion of cost-utility analyses (CUAs) through the medical literature was examined, documenting visible patterns and determining how they correspond with expectations about the diffusion of process innovations.

Methods: This study used 539 CUAs from a registry. It includes data elements comprising year of publication, the research center in which the study was performed, the clinical area covered by the CUA, and the specific journal. Finally, each paper was assigned to a journal type that could be one of the three categories: health services research, general medicine, or clinical specialty.

Results: When the average number of publications is plotted against time, the plot reveals an S-shaped curve. It appears that, whereas CUAs initially were published more frequently in general medical or health services research journals, there was a clear increase in the diffusion of CUA into subspecialty journals over time. The concentration ratio for research centers as measured by the Herfindhal-Hirschman Index decreased over time.

Conclusions: The spread of CUA through the medical literature follows patterns identified for the diffusion of other new technologies and processes. Future research should focus on what impact this spread has had on the practice of medicine and formulation of health policy.

Keywords: Diffusion, Innovation, Cost-utility analysis

Over the past 25 years, rising medical cost has been a common concern of policy-makers as well as researchers. In the 1970s, methodology intended to assess both the cost and benefits gained from health-care expenditures entered the medical literature. For simplicity, we refer to those cost-effectiveness analyses using quality-adjusted life years (QALYs) as an outcome measure as cost-utility analyses (CUAs).

Cost-utility analysis is a methodological approach to assessing the value of a given health technology, program, or intervention. As such, it can be considered a process innovation, one of many designed to improve the ability to make informed decisions about utilization and coverage of medical interventions. One concern about CUAs is that they have not been broadly used by decision-makers in health care. This finding may be because CUAs have been performed by researchers and may not be broadly accessible to or interpretable by policy-makers $(4 ; 5 ; 11)$. Medical practitioners and policy-makers may be unsure about the correctness of the analyses or its real-life implications for the medical-care 
system $(7 ; 8)$. In addition, the analyses may not be useful because the end goals are constrained by pressures other than simple economic efficiency.

The use of an innovation also depends on the stage of innovation. Typologies for the stage of innovation have been described previously. Generally, innovation begins with realization of the need for innovation. A second stage enacts ideas for the specific form of the innovation. In the third stage, the innovation is first brought into practical use. A final stage includes the routine use of the innovation (9). The recommendation of the Panel on Cost-Effectiveness Analysis in Health and Medicine led to the enactment of a plan for a specific form of cost-utility analysis (referred to as a "reference case") so that analyses would be comparable across different medical practices and disease types (3). Since that time, some organizations have begun to use CUA more broadly in decision making $(2 ; 10)$. In addition to implementation, innovation faces the question of diffusion or dissemination. Potential users of an innovation need to become aware of the innovation and then make a decision about whether or not to adopt it. Individuals and organizations can be considered along an adoption continuum starting with the innovators and progressing from early adopters to those who wait until the technology is well-developed and understood (1;9). Successful implementation in one setting does not necessarily ensure broad dissemination of an innovation. Our specific interest in this article was to examine the diffusion of CUAs through the medical literature to determine what patterns can be seen and how they correspond with general expectations about the diffusion of process innovations.

\section{METHODS}

A systematic review of the English-language medical literature from 1976 through 2001 identified 539 CUAs that met inclusion criteria for this study published in MEDLINE-indexed journals (http://www.hsph. harvard.edu/cearegistry/refs.html). Each article was read independently by two readers using a standardized data form, with consensus meetings to resolve discrepancies. For this study, we included data elements encompassing year of publication, the research center in which the study was performed, the clinical area covered by the CUA, and the specific journal. Finally, we assigned each paper to a journal type that could be one of the three categories: health services research (e.g. Journal of Health Services Research), general medicine (e.g. JAMA, Annals of Internal Medicine), or clinical specialty (e.g. Radiology, Journal of Clinical Oncology).

We used the HHI to measure dispersion and share of CUAs performed in various centers. HHI is calculated by squaring the market share (percentage) of each firm (institution) "competing" in the market (of CUAs) and then summing the resulting numbers. We examined these data to determine whether the dissemination of published CUAs followed patterns identified for other innovations and what patterns in clinical areas or journal types existed over time.

\section{RESULTS}

Over the study period, a total of 539 studies were identified and included. The number of cost-utility analyses published on an annual basis rose steadily between 1976 and 1999 and

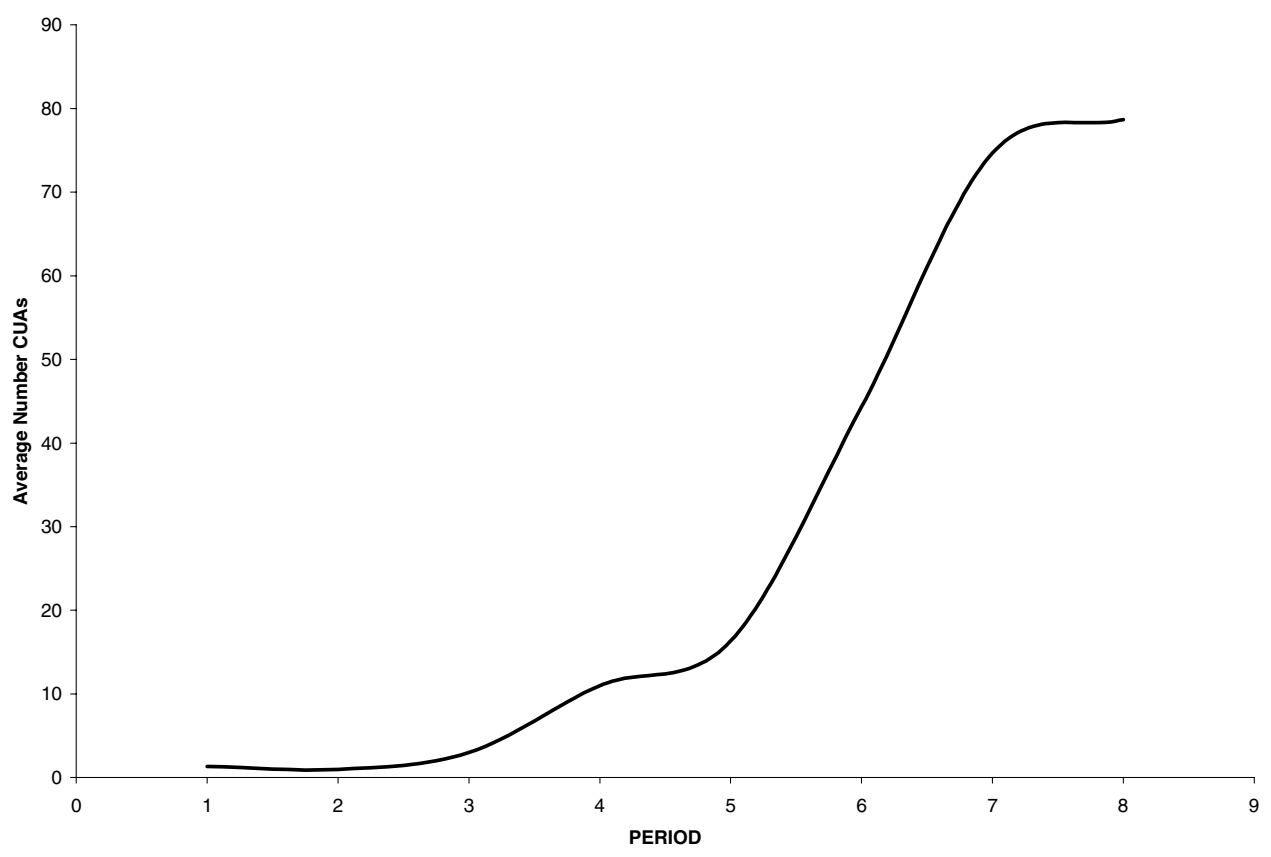

Figure 1. Dissemination curve for cost-utility analyses (CUA) publications. 


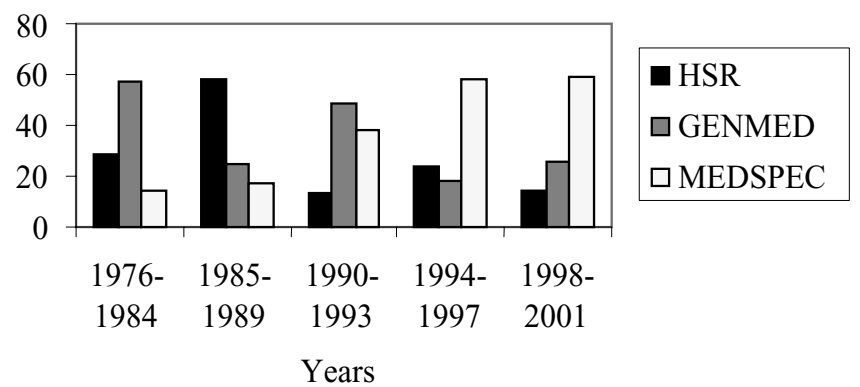

Figure 2. Changes in percentage of articles published in each journal type over time. HSR, health services research; GENMED, general medicine; MEDSPEC, clinical specialty.

then leveled out at approximately 85 publications per year. When the average number of publications is plotted against 3-year periods (Figure 1), the plot reveals an S-shaped curve that parallels curves seen throughout the literature on the diffusion of innovations (6).

The 539 studies appeared in a total of 204 different journals and covered many clinical areas, particularly cardiology, oncology, and pharmacology. The journals themselves included general medical, health services research, and clinical specialties, including pharmacology and nursing. Figure 2 shows the percentage of articles appearing in each journal type over time. It appears that, whereas CUAs were initially published more frequently in general medical or health services research journals, there was a clear increase in the diffusion of CUA into subspecialty journals over time. Table 1 shows dates of first publication of identified CUAs in specific specialties as well as the pattern of publication in that specialty area over time. The concentration ratio for research centers as measured by the HHI decreased over time (Figure 3)

\section{CONCLUSIONS}

The spread of CUA through the medical literature follows patterns identified for the diffusion of other new technologies and processes. Diffusion processes are dependent on perceptions of innovations, characteristics of potential adopters and contextual factors. In the case of CUA, changing healthcare environments leading to improved need for and perception of cost-based studies and the wider training of clinicians and researchers in CUA methodology are likely to have interacted to lead to the diffusion patterns seen in this work.

Early adopters of the CUA innovation were affiliated with very few research institutes, but the concentration ratio has decreased as the innovation has spread to additional centers. As with other new methods and innovations, the next question to answer is what impact this spread has had on the practice of medicine and formulation of health policy.

Table 1. Dates of First Publication of CUAs in Various Specialty Areas and Patterns of Publication in Specialty Journals over Time

\begin{tabular}{|c|c|c|c|c|}
\hline Specialty & First CUA & 1976-1990 & 1991-1996 & 1997-2002 \\
\hline Anesthesia & 1995 & 0 & 1 & 0 \\
\hline Cardiology & 1991 & 0 & 8 & 15 \\
\hline Critical Care & 1996 & 0 & 1 & 4 \\
\hline Dermatology & 1998 & 0 & 0 & 2 \\
\hline Endocrinology & 1994 & 0 & 1 & 5 \\
\hline Gastroenterology & 1991 & 0 & 8 & 15 \\
\hline General Medicine & 1977 & 23 & 33 & 87 \\
\hline Hematology & 1982 & 1 & 7 & 13 \\
\hline Infectious Disease & 1991 & 0 & 1 & 20 \\
\hline Mental Health & 1994 & 0 & 2 & 6 \\
\hline Neurology & 1997 & 0 & 0 & 8 \\
\hline Non-clinical/methods & 1976 & 5 & 19 & 28 \\
\hline Nursing & 1995 & 0 & 1 & 1 \\
\hline Oncology & 1988 & 2 & 10 & 29 \\
\hline Ophthalmology & 2000 & 0 & 0 & 5 \\
\hline Otolaryngology & 1995 & 0 & 2 & 2 \\
\hline Pathology & 1985 & 1 & 0 & 1 \\
\hline Pediatric Medicine & 1993 & 0 & 2 & 5 \\
\hline Pharmacology & 1994 & 0 & 8 & 30 \\
\hline Pulmonary & 1994 & 0 & 4 & 8 \\
\hline Radiology & 1990 & 1 & 6 & 20 \\
\hline Rheumatology & 1994 & 0 & 4 & 8 \\
\hline Surgery & 1993 & 3 & 26 & 22 \\
\hline Urology & 1995 & 0 & 2 & 3 \\
\hline Women's medicine & 2001 & 0 & 0 & 4 \\
\hline
\end{tabular}

CUA, cost-utility analysis. 
Sonnad et al.

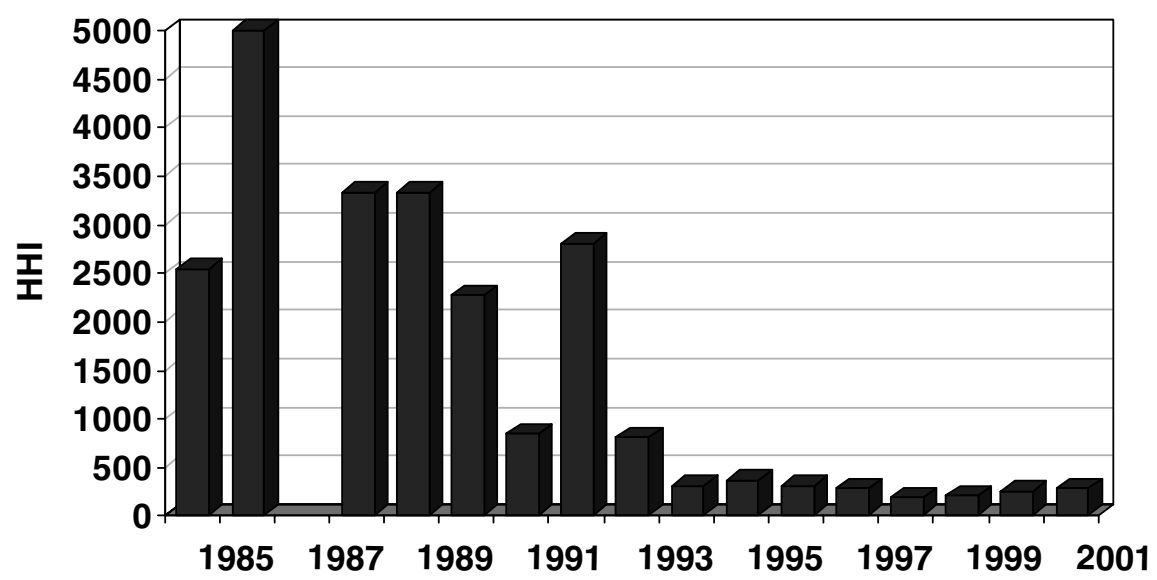

Figure 3. Change in Herfindhal-Hirschman Index $(\mathrm{HHI})$ over time.

\section{CONTACT INFORMATION}

Seema S. Sonnad, PhD, Associate Professor (seema. sonnad@uphs.upenn.edu), Department of Surgery, University of Pennsylvania; Director of Outcomes Research, Department of Surgery, University of Pennsylvania Health System, 4 Silverstein, 3400 Spruce Street, Philadelphia, Pennsylvania 19104-4283, USA

Dan Greenberg, PhD, Senior Lecturer (dangr@bgu.ac.il), Health Systems Management, Ben-Gurion University of the Negev, P.O. Box 653, Beer-Sheva 84105, Israel

Allison B. Rosen, MD, MPH, ScD, Assistant Professor (abrosen@umich.edu), Division of General Medicine, Department of Health Management and Policy, University of Michigan Health System, 300 North Ingalls, Room 7E05, Ann Arbor, Michigan 48109, USA

Peter J. Neumann, ScD (pneumann@hsph.harvard.edu), Department of Health Policy and Management, Harvard School of Public Health, 718 Huntington Avenue, Boston, Massachusetts 02115, USA

\section{REFERENCES}

1. Berwick DM. Disseminating innovations in health care. JAMA. 2003;289:1969-1975.

2. Birch S, Gafni A. The 'NICE' approach to technology as- sessment: An economics perspective. Health Care Manag Sci. 2004; 7:35-41.

3. Gold MR, Siegel JE, Russell LB, Weinstein MC. Costeffectiveness in health and medicine. New York, NY: Oxford University Press; 1996.

4. Hoffmann C, Graf von der Schulenburg JM. The influence of economic evaluation studies on decision making. A European survey. The EUROMET group. Health Policy. 2000;52:179192.

5. Hutton J, Brown RE. Use of economic evaluation in decisionmaking: What needs to change? Value Health. 2002;5:65-66.

6. Mahajan V, Peterson RA. Models for innovation diffusion. Series: Quantitative Applications in the Social Sciences. Beverly Hills: Sage Publications; 1985.

7. Neumann PJ. Why don't Americans use cost-effectiveness analysis? Am J Manag Care. 2004;10:308-312.

8. Prosser LA, Koplan JP, Neumann PJ, Weinstein MC. Barriers to using cost-effectiveness analysis in managed care decision making. Am J Manag Care. 2000;6:173-179.

9. Rogers EM. Diffusion of Innovations. Fourth ed. New York, NY: Free Press.

10. Singer S, Bergthold L, Vorhaus C, et al. Decreasing variation in medical necessity decision making: Final report to the California Health Care Foundation. Stanford, CA: Center for Health Policy, Stanford University; 1999.

11. Sloan FA, Whetten-Goldstein K, Wilson A. Hospital pharmacy decisions, cost containment, and the use of cost-effectiveness analysis. Soc Sci Med. 1997;45:523-533. 